\title{
VIGOR DE LAS SEMILLAS Y PRODUCTIVIDAD DE VARIEDADES DE MAÍZ ${ }^{1}$
}

\author{
Margarita Tadeo-Robledo ${ }^{2}$, Alejandro Espinosa-Calderón ${ }^{3}$, Roberto Valdivia-Bernal ${ }^{4}$,Noel Gómez-Montiel ${ }^{5}$, \\ Mauro Sierra-Macías ${ }^{6}$, Benjamín Zamudio-González.
}

\section{RESUMEN}

Vigor de las semillas y productividad de variedades de maíz. En Cuautitlán Izcalli, México (FESC-UNAM), en el ciclo primavera verano del 2004, con el objetivo de determinar el efecto de la época de cosecha en el vigor de la semilla de maíz, se evaluó la productividad de cuatro variedades de maíz amarillo de ciclo precoz, cosechadas en cinco épocas, las semillas se almacenaron hasta la primavera - verano de 2007. En dos de las variedades, se midió la germinación y vigor, así como las diferencias en el vigor por tamaño de semilla (pequeña y grande menor a $7 \mathrm{~mm}$ o mayor a $7 \mathrm{~mm}$, respectivamente) en cada época de cosecha. Para cada genotipo se estimó el porcentaje de materia seca, de grano, y el rendimiento. Se empleó en el campo un diseño bloques completos al azar con tres repeticiones, para la distribución de los tratamientos, el análisis estadístico fue en forma factorial, se consideraron los factores: genotipos, épocas de cosecha, la interacción genotipos x épocas de cosecha. Los mayores rendimientos $(6541 \mathrm{~kg} / \mathrm{ha}, 7335 \mathrm{~kg} / \mathrm{ha}$ y $7550 \mathrm{~kg} / \mathrm{ha}$ ) se obtuvieron en la cosecha efectuada a los 149 , 152 y 156 días después de la siembra, cuando las variedades estaban en la madurez fisiológica. Las tres variedades de textura cristalina: Oro Ultra 1C (6477 kg/ha), Oro Ultra $2 \mathrm{C}$ (6242 kg/ha), Oro Ultra 3C (6210 kg/ha), fueron diferentes estadísticamente a Oro Plus 1D (4575 kg/ha), de textura dentada. No se encontraron diferencias significativas en el vigor entre variedades. La semilla grande mostró el mayor peso seco de la plántula.

Palabras clave: Zea mays, variedades cristalinas, variedades dentadas, madurez fisiológica.

\begin{abstract}
Seed vigor and productivity of maize varieties. In Cuautitlán Izcalli, Mexico (FESC-UNAM), during the 2004 spring-summer season, in order to determine the effect of harvest time on the vigor of maize seeds, the productivity of four early-maturing, yellow-grain maize varieties, harvested at five different times and stored until spring-summer 2007 was determined. For two varieties, seed germination and vigor were measured, as well as the differences in vigor due to seed size (small and large, smaller than or larger than 7 $\mathrm{mm}$, respectively) during each harvest time. The percentage of dry matter, grain ratio and yield were measured for the four genotypes. In the field, a randomized complete block design with three replications was used for treatment distribution. Statistical analysis was performed as a factorial, considering the factors genotype, harvesting dates, and the interaction genotype $\mathrm{x}$ harvesting date. The highest yields (6541 kg/ha, $7335 \mathrm{~kg} / \mathrm{ha}$ and $7550 \mathrm{~kg} / \mathrm{ha})$ were obtained at harvesting dates of 149, 152 and 156 days after planting, when the varieties had reached physiological maturity. The three maize varieties with flint grain: golden ultra 1C (6477 $\mathrm{kg} / \mathrm{ha}$ ), Golden Ultra 2C (6242 kg / ha), and Golden Ultra 3C $(6210 \mathrm{~kg} / \mathrm{ha}) \mathrm{had}$ better yield than the variety Gold Plus 1D (4575 kg/ha), with dented grain. No differences in seed vigor were detected in the varieties studied. Larger seeds produced seedlings with higher dry weight.
\end{abstract}

Key words: Zea mays, flint varieties, dent varieties, physiological maturity.

\footnotetext{
Recibido: 22 de marzo, 2009. Aceptado: 17 de mayo, 2010. Investigación realizada dentro del Programa de Apoyo a Proyectos de Investigación e Innovación Tecnológica (PAPIIT IN 205908) de la Universidad Nacional Autónoma de México (UNAM).

2 Ingeniería Agrícola, Facultad de Estudios Superiores Cuautitlán - Cuautitlán, Universidad Nacional Autónoma de México (UNAM), km. 2.5 Carretera Cuautitlán-Teoloyucan, C.P. 54700. Apdo. Postal 25, Cuautitlán, Izcalli, Edo. de México. tadeorobledo@yahoo.com

3 Campo Experimental Valle de México, Instituto Nacional de Investigaciones Forestales, Agrícolas y Pecuarias (INIFAP), Km. 18.5 Carr. México - Lechería, Apdo. Postal 10, C.P. 56230, Chapingo, México. Tel. (595) 9542877 extensión 119. (Autor para correspondencia). espinoale@yahoo.com.mx

4 Universidad Autónoma de Nayarit, Tepic, Nayarit, México. beto49_2000@yahoo.com.mx

5 Campo Experimental Iguala, Instituto Nacional de Investigaciones Forestales, Agrícolas y Pecuarias (INIFAP), Iguala, Guerrero. México. noelorlando19@hotmail.com

6 Campo Experimental Cotaxtla, Instituto Nacional de Investigaciones Forestales, Agrícolas y Pecuarias (INIFAP), Veracruz, Ver., México.
} 


\section{INTRODUCCIÓN}

En la producción de semillas es importante definir la madurez fisiológica, ya que en este punto, es donde se alcanza el máximo de calidad fisiológica (Copeland y McDonald 2001), y el mayor nivel en acumulación de materia seca, ocurre en la madurez fisiológica, misma que se ubica cercana a 35\% de humedad en la semilla (Copeland y McDonald 2001, Perry 1981a, Perry 1981 b, Perry 1983, McDonald y Copeland 1997).

En la Facultad de Estudios Superiores Cuautitlán, UNAM, en años anteriores se han desarrollado variedades de polinización libre de maíz, para los Valles Altos de México (2200 a 2600 msnm). Entre ellos Oro Ultra y Oro Plus (Tadeo et al. 2004, Espinosa et al. 2005, Tadeo et al. 2005a, Tadeo et al. 2005b, Tadeo et al. 2005c), las cuales son de ciclo precoz, con adaptación a siembras tardías, en temporal retrasado. Estas variedades han mostrado ventajas como tolerancia al acame, representando una opción ya que no hay variedades mejoradas de grano amarillo en uso comercial actualmente. En el pasado se liberaron las variedades Amarillo Zanahoria por parte de ICAMEX y la variedad V-26 A, por el INIFAP (Gamez et al. 1996).

La mejor época de cosecha, se ubica en la madurez fisiológica o posterior a ella, dependiendo de la infraestructura que se disponga para el secado y su ciclo de siembra a madurez fisiológica, o con base en las unidades térmicas. Se trata de relacionar las épocas de cosecha con el vigor de la semilla cosechada, ya que el vigor es una característica relevante en siembras comerciales, para lograr un adecuado establecimiento de plantas en condiciones limitantes o desfavorables (McDonald y Copeland 1997). Conviene establecer la época más adecuada de cosecha, posterior a esta madurez fisiológica, que depende de la disponibilidad de infraestructura para secado, entre más cercana esté la cosecha de esta fecha, combinado con un buen secado se preserva la calidad.

El objetivo de este trabajo fue determinar el efecto de la época de cosecha en el vigor de la semilla de maíz.

\section{MATERIALES Y MÉTODOS}

El trabajo de campo se llevó a cabo durante el ciclo primavera-verano 2004, en el Rancho Almaraz, de la Facultad de Estudios Superiores Cuautitlán, de la Universidad Nacional Autónoma de México (FESC, UNAM), ubicado a $30 \mathrm{~km}$ al norte de la Ciudad de México, a una altitud de $2274 \mathrm{~m}$, la cual se encuentra delimitada por los paralelos $19^{\circ} 39^{\prime}-19^{\circ} 45^{\prime}$ latitud norte y los meridianos $99^{\circ} 88^{\prime}-9^{\circ} 14^{\prime}$ ' longitud oeste. Se establecieron lotes de producción de semilla de cuatro variedades de maíz amarillo de ciclo precoz (Oro Plus, Oro Ultra 1C, Oro Ultra 2C, Oro Ultra 3C), la fecha de siembra fue el 12 de junio del 2004, para los cuatro genotipos. Las fechas de cosecha, en días después de la siembra, fueron: a los 129 días (19 de octubre), 135 días (25 de octubre), 149 días (8 de noviembre), 152 días (11 de noviembre), y a los 156 días (15 de noviembre).

Se empleó en el campo experimental un diseño de bloques completos al azar con tres repeticiones, el análisis estadístico fue en forma factorial para: variedad, épocas de cosecha, la interacción variedad $\mathrm{x}$ épocas de cosecha. En cada variedad se estimó porcentaje de materia seca, de grano, el peso hectolitrito, longitud de mazorca, las hileras por mazorca, los granos por hilera, así como el rendimiento.

Después de la cosecha, la semilla se almacenó hasta el año 2007. Se evaluó el vigor, en dos de las variedades, en las instalaciones de la Facultad de Estudios Superiores Cuautitlán (FES Cuautitlán-UNAM), en el invernadero y en el laboratorio de Producción y Tecnología de semillas. La FESC se localiza a $30 \mathrm{~km}$, al norte de la Ciudad de México, delimitada por los paralelos $19^{\circ} 39^{\prime}-19^{\circ} 45^{\prime}$ latitud $\mathrm{N}$ y los meridianos 99 $88^{\prime}$ - $99^{\circ} 14^{\prime}$ longitud $\mathrm{W}$, a una altitud de 2274 msnm.

De acuerdo a la clasificación de Köpen adaptada a las condiciones de México por Enriqueta García (1981), el clima de Cuautitlán se clasifica como C(W0)(W)b (i"), denominado templado subhúmedo, el más seco de los templados sub-húmedos, con una temperatura media anual de $14,8^{\circ} \mathrm{C}$ con un régimen de lluvia en verano y menos del $5 \%$ de lluvias en invierno, poca variación térmica y una precipitación anual de 609,2 $\mathrm{mm}$.

Las variedades Oro Plus, Oro Ultra 1C, Oro Ultra 2C, Oro Ultra 3C, poseen adaptación a Valles Altos (2200 a $2600 \mathrm{msnm}$ ), con ciclo vegetativo 15 días más precoces que H-33 y VS-22. Su altura de planta y mazorca es inferior a $200 \mathrm{~cm} \mathrm{y} 100 \mathrm{~cm}$, respectivamente. La floración masculina se inició a 
los 70 días y la madurez fisiológica a los 135 días, en sitios en altitudes de 2240 metros (Tadeo y Espinosa 2004, Tadeo et al. 2005 a, Tadeo et al. 2005 b, Tadeo et al. 2007).

Para la evaluación de vigor, el diseño utilizado fue el de bloques completos al azar, y los tratamientos fueron la combinación de las dos variedades (Oro Plus 2D y Oro Ultra 3C), dos tamaños de semilla (grande I y pequeña II); así como las interacciones de cada uno de los factores (variedades x tamaños de semi1la). Las variables evaluadas y que permiten definir el vigor, como la condición activa y sana que le permite una germinación uniforme y un rápido crecimiento de la plántula bajo condiciones generales de campo (Perry 1981a, Perry 1981b, Perry 1981c, Perry 1984, Copeland y McDonald 1997), fueron: Velocidad de emergencia, para la cual se llevó a cabo con conteos diarios del número de plantas emergidas por surco, una vez que se inició la emergencia, ocurrió a los siete días después de la siembra. Con base en estos valores de emergencia se aplicó la fórmula que se detalla posteriormente. Longitud de raíz y plúmula, se obtuvo midiendo en $\mathrm{cm}$ la longitud de la raíz desde la inserción, de cinco plántulas elegidas al azar por surco; así como la longitud de la plúmula de cada una de las plántulas extraídas. Peso fresco de raíz y plúmula, este dato se obtuvo con base en un corte con un bisturí de la plántula a partir de la inserción, para separar raíz y plúmula, y pesarla en una balanza granataria. Peso seco de la raíz y de la plúmula, se obtuvo al colocar las partes aéreas y raíces de cada tratamiento en bolsas de papel, y secarlas en una estufa Felisa a una temperatura de $70{ }^{\circ} \mathrm{C}$ durante 72 horas. Una vez que se hubieron deshidratado, se procedió a pesar raíz y plúmula en una balanza granataria, para obtener el peso en gramos.

Se clasificaron las semillas en cada época de cosecha, y se procedió a desgranar y separar por tamaño con base en el empleo de cribas. Para la semilla pequeña, la criba fue de $7 \mathrm{~mm}$, esta medida es utilizada para definir semilla pequeña en trabajos de investigación y por empresas que la comercializan en México. Semilla grande, fue la que no pasó esa criba. Una vez clasificada por tamaño en pequeña y grande, se seleccionaron lotes de 25 semillas y se rotularon los sobres para contenerlas.

Antes de establecer la siembra se limpió el área de siembra de residuos de la cosecha anterior y se removieron $15 \mathrm{~cm}$ de suelo de tipo franco arcilloso.
Este suelo fue cernido fuera de la cama, la capa de suelo que quedó dentro de ésta fue removida, mullida y nivelada. Se utilizó una cama de 1,25 x 13 metros aproximadamente.

La siembra se estableció con el suelo seco el día $1^{\circ}$ de junio del año 2007, de forma manual con una "plantilla"; se distribuyeron 25 semillas a lo ancho de la cama, con una separación de $5 \mathrm{~cm}$ entre ellas. A lo largo de la cama se sembraron 100 surcos con una distancia de $13 \mathrm{~cm}$ entre ellos. Una vez que se colocaron todas las semillas con la corona hacia arriba, se procedió a cubrirlas con una capa de tierra cernida de $10 \mathrm{~cm}$ de espesor.

El primer riego se aplicó después de la siembra, para que la humedad alcanzara a llegar al fondo de la cama, posteriormente se regó diariamente. La extracción de plantas se llevó a cabo a los 25 días después de la siembra. Se aflojó la tierra con la ayuda de una pala plana y se extrajo planta por planta, cuidando que no se dañara el sistema radicular, posteriormente fueron lavadas para remover la tierra adherida a la raíz y se colocaron en sobres.

En el laboratorio se midieron y pesaron para iniciar el proceso de evaluación de las siguientes variables:

La velocidad de emergencia se midió con base en el conteo diario del número de plantas emergidas por surco, una vez que se inició la emergencia, ocurriendo esto siete días después de la siembra. Este dato se calculó mediante la siguiente expresión:

$$
\begin{aligned}
\text { V.E. }=\left(\mathrm{X}_{1}\right) / 1+\left(\mathrm{X}_{2}\right) / 2 & +\left(\mathrm{X}_{3}\right) / 3+\ldots \ldots \ldots . .+\left(\mathrm{X}_{1-1}\right) / \mathrm{n}-1 \\
& +(\mathrm{Xi}) / \mathrm{n}
\end{aligned}
$$

Donde:

$\mathrm{X}=$ número de plántulas emergidas por día $\mathrm{n}=$ número de días después de la siembra $\mathrm{i}=1,2,3, \ldots \mathrm{n}-1, \mathrm{n}$

La longitud de raíz y plúmula, se midió en centímetros con la ayuda de una regla. La longitud de la raíz desde la inserción se obtuvo en cinco plántulas elegidas al azar por surco; así como la longitud de la plúmula de cada una de las plántulas extraídas.

El peso fresco de raíz y plúmula se obtuvieron con un corte de la plántula a partir de la inserción, para separar raíz y plúmula, y posteriormente pesando en una balanza granataria. 
Para obtener el peso seco de raíz y plúmula, se colocaron las partes aéreas y las raíces en bolsas de papel, para posteriormente secarlas en una estufa Felisa a una temperatura de $70{ }^{\circ} \mathrm{C}$ durante $72 \mathrm{~h}$. Una vez que se hubieron deshidratado se procedió a pesar raíz y plúmula en una balanza granataria.

\section{RESULTADOS Y DISCUSIÓN}

En el rendimiento se encontraron diferencias altamente significativas entre genotipos, épocas de cosecha, pero no para la interacción entre estos factores. El coeficiente de variación fue $23,4 \%$ y la media de rendimiento $5875 \mathrm{~kg} / \mathrm{ha}$. Para genotipos se encontraron diferencias altamente significativas para las variables hileras por mazorca y granos por hilera y significativas para porcentaje de materia seca, longitud, diámetro de mazorca y granos por mazorca. En las épocas de cosecha se detectaron diferencias al 0,01 de probabilidad para las variables porcentaje de materia seca, hileras por mazorca, granos por hilera, así como al 0,05\% de probabilidad para porcentaje de grano, longitud de mazorca; en la interacción hubieron diferencias altamente significativas para las variables hileras por mazorca y granos por hilera.

En la comparación de medias para genotipos, las tres variedades de textura cristalina denominadas Oro Ultra 1C (6477 kg/ha), Oro Ultra 2C (6242 kg/ha), Oro Ultra 3C (6210 kg/ha), se ubicaron en el mismo grupo de significancia, y superó estadísticamente a Oro Plus 1D (4575 kg/ha), de textura dentada (Cuadro 1), lo que coincide con resultados anteriores (Tadeo et al. 2004, Tadeo et al. 2005c, Tadeo et al. 2006).

En la comparación de medias para épocas de cosecha, los mejores rendimientos se obtuvieron cuando se cosechó a los 149 días $(6541 \mathrm{~kg} / \mathrm{ha}), 152$ días (7335 $\mathrm{kg} / \mathrm{ha})$ y 156 días (7550 kg/ha) después de la siembra, éstos fueron estadísticamente superiores al obtenido en la época de cosecha a los 135 días (5164 kg/ha) y 129 días $(2788 \mathrm{~kg} / \mathrm{ha})$. Los porcentajes de materia seca, en las primeras épocas de cosecha, 129 días $(24,5 \%)$ y 135 días $(44,1 \%)$, indican que las variedades no habían llegado a la madurez fisiológica (Cuadro 2). En cambio en las épocas de cosecha a los 149,152 y 156 días, los valores de materia seca $(67,1 \%, 71,2 \%$ y $77,2 \%$, respectivamente), confirman que los mayores rendimientos estaban relacionados con la madurez fisiológica, como lo señalan diversos autores (Copeland y McDonald 2001, Perry 1981 a, Perry, 1981 b, Perry 1984, McDonald y Copeland 1997). La información obtenida permite señalar que las mejores fechas de cosecha son aquellas posteriores a la madurez fisiológica.

En la cosecha a partir de la madurez fisiológica se lograron mayores rendimientos, con respecto a la cosecha cuando aún no se lograba este punto fenológico, lo que indica la relevancia de definir con cierta certidumbre esta condición, para asegurar la producción de semilla, por otra parte las tres variedades de textura cristalina: Oro Ultra 1C (6477 $\mathrm{kg} / \mathrm{ha})$, Oro Ultra 2C (6242 kg/ha), Oro Ultra 3C $(6210 \mathrm{~kg} / \mathrm{ha})$, se ubicaron en la media de las diferentes épocas de cosecha en el mismo grupo superior de

Cuadro 1. Comparación de medias de variables evaluadas en cuatro genotipos de maíz cosechados en cinco épocas de cosecha. Facultad de Estudios Superiores Cuautitlán, Cuautitlán Izcalli, México. Primavera - verano, 2004.

\begin{tabular}{lccccccccc}
\hline Variedad & $\begin{array}{c}\text { Rendi- } \\
\text { miento } \\
(\mathbf{k g} / \mathbf{h a})\end{array}$ & $\begin{array}{c}\text { Materia } \\
\text { Seca } \\
\mathbf{( \% )}\end{array}$ & $\begin{array}{c}\text { Por- } \\
\text { centaje } \\
\text { grano }\end{array}$ & $\begin{array}{c}\text { Peso } \\
\text { volumétri- } \\
\mathbf{c o}(\mathbf{k g} / \mathbf{h l})\end{array}$ & $\begin{array}{c}\text { Longitud } \\
\text { mazorca } \\
(\mathbf{c m})\end{array}$ & $\begin{array}{c}\text { Hileras } \\
\text { mazorca }\end{array}$ & $\begin{array}{c}\text { Granos } \\
\text { hilera }\end{array}$ & $\begin{array}{c}\text { Diámetro } \\
\text { mazorca } \\
(\mathbf{c m})\end{array}$ & $\begin{array}{c}\text { \# Granos } \\
\text { por } \\
\text { mazorca }\end{array}$ \\
\hline Oro Plus & $4575 \mathrm{~b}$ & 52,3 & 76,6 & 683 & $15,0 \mathrm{~b}$ & $14,0 \mathrm{~b}$ & $28 \mathrm{bc}$ & 4,5 & 391 \\
Oro Ultra IC & $6477 \mathrm{a}$ & 59,1 & 77,9 & 688 & $15,9 \mathrm{ab}$ & $16,2 \mathrm{a}$ & $26 \mathrm{c}$ & 4,7 & 403 \\
Oro Ultra 2C & $6242 \mathrm{a}$ & 58,4 & 77,5 & 692 & $16,3 \mathrm{ab}$ & $13,9 \mathrm{~b}$ & $30 \mathrm{a}$ & 4,6 & 419 \\
Oro Ultra 3C & $6210 \mathrm{a}$ & 57,4 & 77,9 & 696 & $16,5 \mathrm{a}$ & $14,2 \mathrm{~b}$ & $30 \mathrm{a}$ & 4,5 & 421 \\
\hline D.S.H. $(0,05)$ & 1349 & 7 & 3,7 & 23 & 1,3 & 1,6 & 2 & 0,2 & 36 \\
\hline
\end{tabular}

Medias con la misma letra en las columnas, son iguales estadísticamente (Tukey, 0,05). 
Cuadro 2. Comparación de las medias de las variables evaluadas en cinco variedades de maíz de grano amarillo, durante cinco épocas de cosecha. Facultad de Estudios Superiores Cuautitlán, Cuautitlán Izcalli, México. Primavera - verano. 2004.

\begin{tabular}{lccccccccc}
\hline Época de cosecha & $\begin{array}{c}\text { Rendimien- } \\
\text { to }(\mathbf{k g} / \mathbf{h a})\end{array}$ & $\begin{array}{c}\text { Materia seca } \\
(\boldsymbol{\%})\end{array}$ & $\begin{array}{c}\text { Grano } \\
(\boldsymbol{\%})\end{array}$ & $\begin{array}{c}\text { Peso } \\
\text { volu- } \\
\text { métrico } \\
(\mathbf{K g} / \mathbf{h l})\end{array}$ & $\begin{array}{c}\text { Longitud } \\
\text { mazorca } \\
(\mathbf{c m})\end{array}$ & $\begin{array}{c}\text { Hileras/ } \\
\text { mazorca }\end{array}$ & $\begin{array}{c}\text { Granos/ } \\
\text { hilera }\end{array}$ & $\begin{array}{c}\text { Diámetro/ } \\
\text { Mazorca } \\
(\mathbf{c m})\end{array}$ & $\begin{array}{c}\text { Granos/ } \\
\text { mazorca }\end{array}$ \\
\hline 19 octubre 2004 & $2788 \mathrm{c} *$ & $24,5 \mathrm{~d}$ & $75,0 \mathrm{~b}$ & 689 & 16,4 & $14 \mathrm{~b}$ & $30 \mathrm{a}$ & 4,5 & 412 \\
25 octubre 2004 & $5164 \mathrm{~b}$ & $44,1 \mathrm{c}$ & $76,4 \mathrm{ab}$ & 678 & 16,0 & $14 \mathrm{~b}$ & $29 \mathrm{a}$ & 4,6 & 410 \\
8 noviembre 2004 & $6541 \mathrm{ab}$ & $67,1 \mathrm{~b}$ & $78,9 \mathrm{ab}$ & 692 & 16,4 & $14 \mathrm{~b}$ & $30 \mathrm{a}$ & 4,6 & 423 \\
11 noviembre 2004 & $7335 \mathrm{a}$ & $71,2 \mathrm{ab}$ & $76,5 \mathrm{ab}$ & 694 & 14,9 & $17 \mathrm{a}$ & $24 \mathrm{~b}$ & 4,5 & 393 \\
15 noviembre 2004 & $7550 \mathrm{a}$ & $77,2 \mathrm{a}$ & $80,5 \mathrm{a}$ & 695 & 15,8 & $14 \mathrm{~b}$ & $29 \mathrm{a}$ & 4,5 & 404 \\
\hline D.S.H. $(0,05)$ & 1608 & 8,5 & 4,5 & 28 & 1,6 & 2 & 2 & 0,2 & 43 \\
\hline
\end{tabular}

* Medias con la misma letra en las columnas, son iguales estadísticamente (Tukey $(0,05)$.

significancia, superando estadísticamente a Oro Plus 1D $(4575 \mathrm{~kg} / \mathrm{ha})$, de textura dentada.

Con base en las cuatro variedades y cinco diferentes muestras de semilla, es decir las fechas de cosecha, se eligieron dos variedades (Oro Ultra $3 \mathrm{C}$ y Oro Plus 2D), así como semilla de cada una de ellas cosechada en madurez fisiológica, se tomaron muestras de cada una de las dos variedades y dos tamaños de semilla (grande y pequeña), con las cuales se efectuó un ensayo de vigor.

El ensayo de vigor se estableció en un diseño de bloques al azar, el análisis factorial consideró los tratamientos variedades, así como tamaños de semilla y la interacción entre variedades y tamaños de éstas. Los resultados mostraron que para variedades en la variable velocidad de emergencia se detectó diferencia altamente significativa, no así para tamaño de semilla, tampoco para la interacción variedades x tamaño de semilla. Para peso fresco de raíz se detectó diferencia significativa en la interacción variedades $\mathrm{x}$ tamaño de semilla. En la variable peso seco de plántula se observó diferencia significativa al nivel de 0,05 de probabilidad, para tamaño de semilla.

En la comparación de medias entre las dos variedades en estudio (Oro Ultra 3C y Oro Plus 2D) para las diferentes variables: días a emergencia, longitud de la plúmula, longitud de la radícula, peso fresco de la plántula y de la raíz, peso seco de la plántula y de la raíz, en todos los casos, no hubo diferencias significativas, lo que señala que los dos tamaños de semilla presentaron similar respuesta en estas variables que miden el vigor, lo que podría tener su explicación en que la profundidad de siembra, tipo de suelo y humedad del suelo disponible, fueron condiciones favorables, no ofrecieron condiciones adversas, como mayor profundidad de siembra, suelo pesado, limitación de humedad de suelo, que propiciaran una diferente expresión de vigor (ISTA 2001, Copeland y McDonald 2001).

Cuando se compararon las medias obtenidas entre los dos tamaños de semilla evaluados con respecto a la expresión de vigor medida con diferentes variables, se observa que para el peso fresco de raíz y peso seco de plántula, hubo dos grupos de significancia, en cambio en las otras variables evaluadas se presentó un sólo grupo de significancia, lo que confirma que las condiciones de suelo, humedad y profundidad de siembra fueron favorables y no permitieron expresar diferencias en vigor.

En el Cuadro 3, se presentan los valores medios obtenidos para cada una de las dos variedades en combinación con tamaño grande y pequeño de semilla y los valores medios de los resultados obtenidos de cada una de las variedades en todas las fechas de cosecha, para las variables rendimiento y peso volumétrico (Figura 1) y materia seca y longitud de mazorca (Figura 2).

No se encontró diferencia significativa en las variables de vigor entre las variedades, sin embargo hubo diferencia altamente significativa en el peso seco de la plúmula en los tamaños de semilla, el mejor se obtuvo con el tamaño grande. Finalmente se observó diferencia significativa en el peso fresco de la raíz en la interacción del tamaño de semilla por variedad. 
Cuadro 3. Comparación de medias de variables de vigor evaluadas en dos variedades de maíz (Oro Ultra 3C y Oro Plus 2D) y dos tamaños de semilla (grande (mayor de $7 \mathrm{~mm}$ ) y pequeña (menor de $7 \mathrm{~mm}$ )). Facultad de Estudios Superiores Cuautitlán, Cuautitlán Izcalli, México. Primavera verano 2007.

\begin{tabular}{lcccc}
\hline & \multicolumn{3}{c}{ Variedad } \\
\cline { 2 - 5 } \multicolumn{1}{c}{ Variable } & \multicolumn{2}{c}{ Oro Ultra } & \multicolumn{2}{c}{ Oro Plus } \\
\cline { 2 - 5 } & \multicolumn{2}{c}{ tamaño semilla } & tamaño semilla \\
\cline { 2 - 5 } & grande & 5,07 & 4,45 & pequeña \\
\hline Velocidad emergencia & 4,14 & 30,7 & 31,09 & 5,12 \\
Longitud de plántula (cm) & 32,81 & 15,05 & 16,88 & 29,16 \\
Longitud raíz (cm) & 17,11 & 13,43 & 13,02 & 14,71 \\
Peso fresco plántula $(\mathrm{g})$ & 13,76 & 3,06 & 3,32 & 10,60 \\
Peso fresco raíz $(\mathrm{g})$ & 3,65 & 0,55 & 0,73 & 2,31 \\
Peso seco plántula $(\mathrm{g})$ & 0,67 & 1,53 & 1,55 & 0,45 \\
Peso seco raíz $(\mathrm{g})$ & 1,43 & & & 1,17 \\
\hline
\end{tabular}

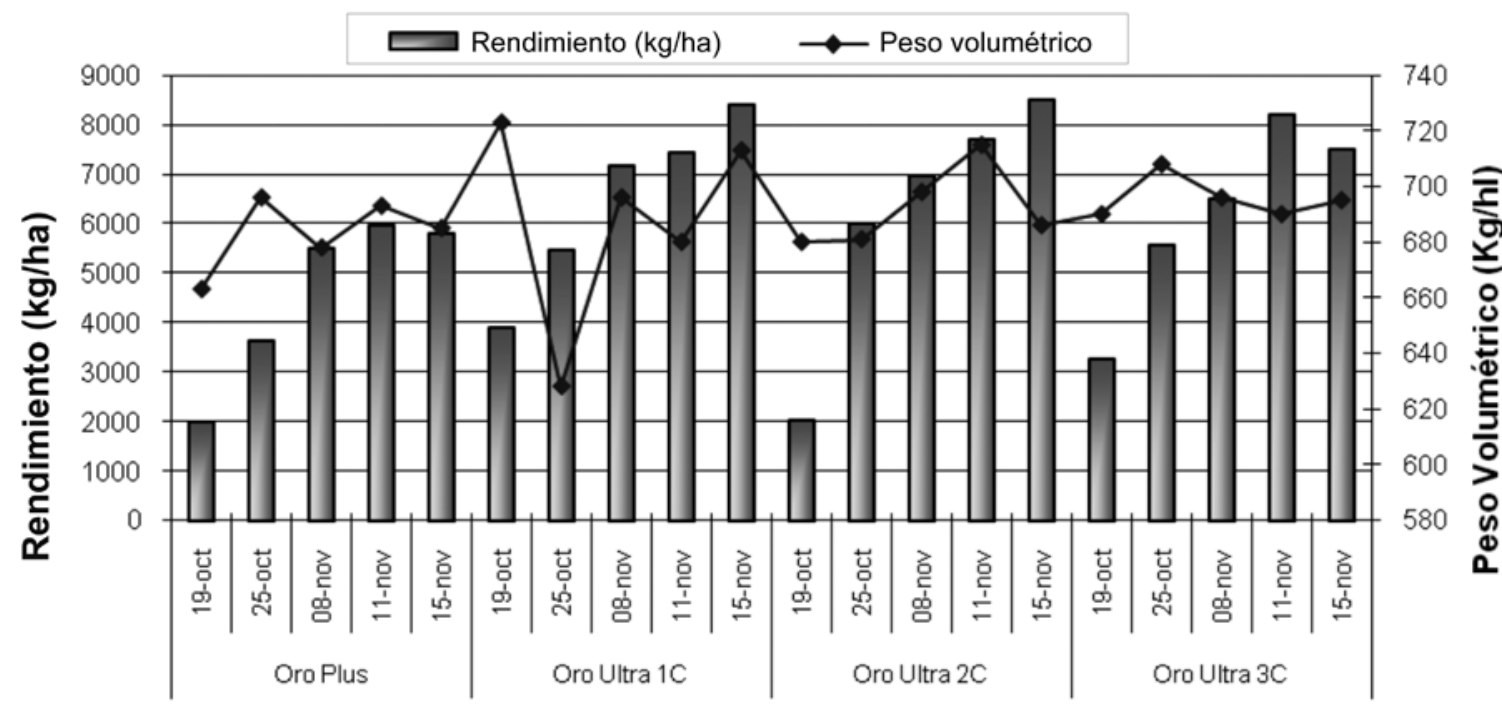

Época de cosecha/variedad

Figura 1. Medias de rendimiento $(\mathrm{kg} / \mathrm{ha})$ y peso volumétrico $(\mathrm{kg} / \mathrm{hl})$ de la semilla de cuatro variedades de maíz cosechadas en cinco diferentes fechas. Facultad de Estudios Superiores Cuautitlán, Cuautitlán Izcalli, México. Primavera - Verano 2004. 


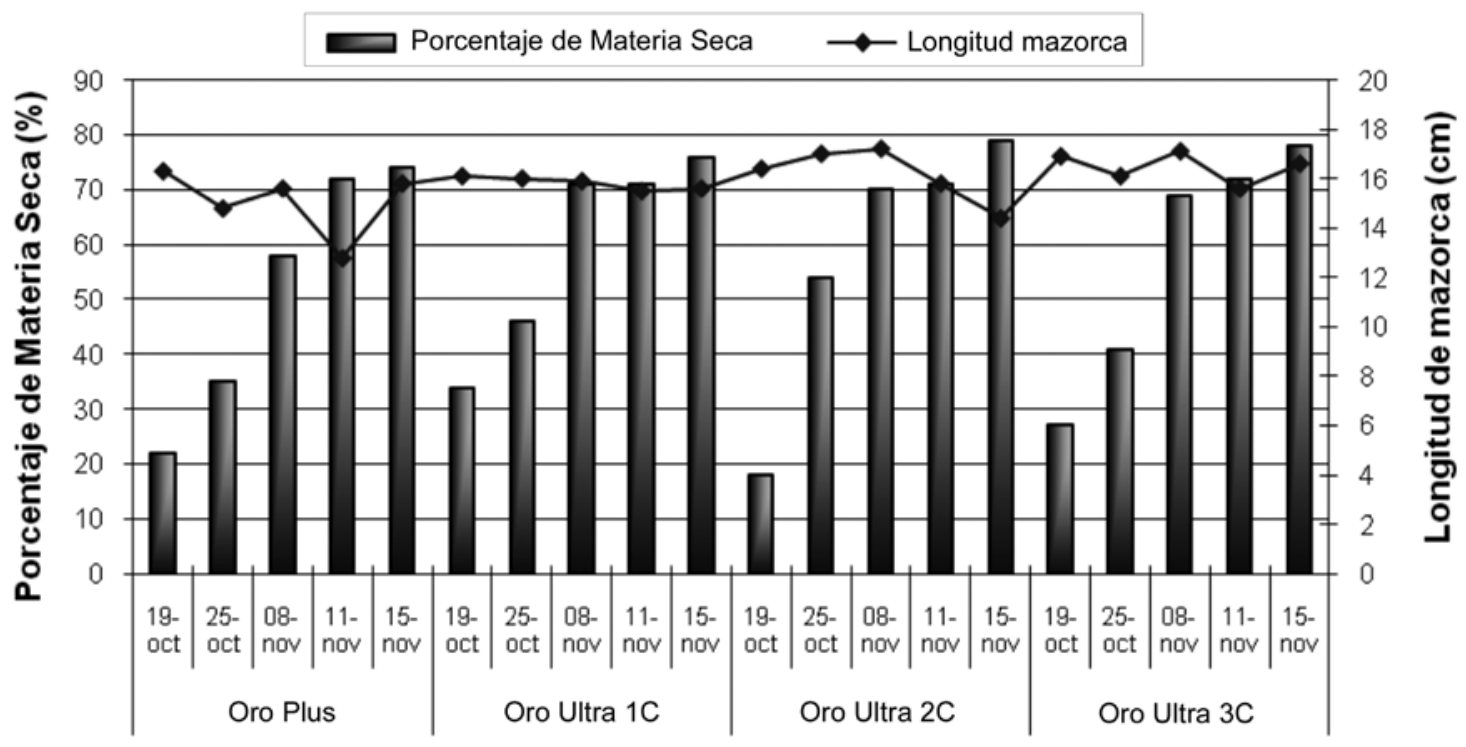

\section{Época de cosecha/variedad}

Figura 2. Medias de porcentaje de materia seca y longitud de mazorca $(\mathrm{cm})$ de la semilla en cuatro variedades de maíz cosechadas en cinco diferentes fechas. Facultad de Estudios Superiores Cuautitlán, Cuautitlán Izcalli, México. Primavera - Verano 2004.

\section{AGRADECIMIENTOS}

Los autores agradecen el apoyo financiero del Programa de Apoyo a Proyectos de Investigación e Innovación Tecnológica (PAPIIT IN 205908) de la Universidad Nacional Autónoma de México (UNAM), para la realización de la presente investigación.

\section{LITERATURA CITADA}

Copeland, LO; McDonald, MB. 2001. Principles of seed science technology. 4 ed. Burguess Publishing Company. Mineapolis, Minnesota, USA. p. 121-144.

Espinosa, A; Tadeo, M; Martínez, R. 2005. Nuevas variedades de maíz de grano amarillo para Valles Altos de México generados en la UNAM. Agrosíntesis. p. $17-21$.

Gamez, VJA; Ávila, PMA; Angeles, AH; Díaz, HC; Ramírez, VH; Alejo, JA; Terrón, IA. 1996. Híbridos y variedades de maíz liberados por el INIFAP hasta 1996. INIFAP, SAGAR. Publicación Especial No. 16. Toluca, México. $102 \mathrm{p}$.

García, E. 1981. Modificaciones al sistema de clasificación climática de Köppen: para adaptarlo a las condiciones de la república mexicana. Dirección General de Publicaciones. UNAM. México, D. F. 252 p.

ISTA (International Seed Testing Association). 2001. International rules for seed testing 2001. Seed Sci. and Thecnol. 29, Supplement 2. 28-29 p.

McDonald, MB; Copeland, LO. 1997. Seed production principles and practices. Chapman \& Hall. New York, USA. $749 \mathrm{p}$.

Perry, DA. 1981a. Introduction. In: Perry, DA. ed. Handbook of vigour test methods. International Seed Testing Association. Zurich, Switzerland. p. 8-9.

Perry, DA. 1981b. Methodology and application of vigour test. In: Perry, DA. ed. Handbook of vigour test methods. International Seed Testing Association. Zurich, Switzerland. p. 3-7. 
Perry, DA. 1981c. Seedling growth and seedling evaluation tests. In: Handbook of vigour test methods. Perry, DA. ed. International Seed Testing Association. Zurich, Switzerland. p. 10-20.

Perry, DA. 1984. Commentary on ISTA vigour test committee collaborative trial. Seed Science Technology 12:301-308.

Tadeo, M; Espinosa, A; Martínez, R; Srinivasan, G; Beck, D; Lothrop, J; Torres, JL; Azpiroz, S. 2004. Puma 1075 y Puma 1076 híbridos de maíz de temporal para los Valles Altos de México (2200 a 2600 msnm). Rev. Fitotecnia Mexicana 27(2):211-212.

Tadeo, M; Espinosa, A. 2004. Producción de semilla y difusión de variedades e híbridos de maíz de grano amarillo para Valles Altos de México. Revista FESC Divulgación Científica Multidisciplinaria. Año 4(14):5-10.

Tadeo, M; Espinosa, A; Martínez, R; Solano, AM. 2005 a. Puma 1076 híbrido de maíz para los Valles Altos de México (2200 a $2600 \mathrm{msnm}$ ). Universidad Nacional Autónoma de México, Facultad de Estudios Superiores Cuautitlán, Cuautitlán Izcalli, México. Desplegable técnica 3. p. 3.

Tadeo, M; Espinosa, A; Martínez, R; Solano, AM. 2005 b. Puma 1075 híbrido de maíz para los Valles Altos de México (2200 a $2600 \mathrm{msnm}$ ). Universidad Nacional Autónoma de México, Facultad de Estudios Superiores
Cuautitlán, Cuautitlán Izcalli, México. Desplegable Técnica no. 1. p. 3.

Tadeo, M; Espinosa, A; Martínez, R; Arias, R. 2005 c. Producción y tecnología de semillas, desarrollo y difusión de híbridos y variedades de maíz de la UNAM para su adopción extensiva en México. XX Reunión Latinoamericana de Maíz. Editores Miguel Barandiaran Gamarra, Alexander Chávez Cabrera, Ricardo Sevilla Panizo, Teodoro Narro León. Lima, Perú. p. 435-441.

Tadeo, M; Espinosa, A; Martínez, R; Salazar, D; Téllez, C; Osorio, JM. 2006. Plant breeding and maize seed production at the Agricultural Engineering Department of the National University of Mexico (UNAM)", in: Book of Poster Abstracts. International Plant Breeding Symposium. ed. Sophie Higman, Mexico, City, 20-25 august, 2006. p. 118.

Tadeo, RM; Espinosa, CA; Martínez, MR; Cosme, T; Gonzalez, RI; Osorio, HJM; Valdivia, BR; Gómez, MN; Sierra, MM; Caballero, HF; Palafox, CA; Rodríguez, MF. 2007. Maize seed production and plant breeding in relation with the process teaching - learning at the National Autonomous University of Mexico (UNAM). El Minia, Egypt. African Crop Science Conference Proceedings, African Crop Science Society Egypt 8:19-22. 